\title{
ExoSense: measuring manipulation in a wearable manner
}

\author{
Edoardo Battaglia, Manuel G. Catalano, Giorgio Grioli, Matteo Bianchi and Antonio Bicchi
}

\begin{abstract}
Grasp and manipulation is a complex task, deceivingly simple to accomplish for humans in everyday life, yet challenging to implement in a robotic hand. There is a trend in literature to use information obtained from studies on human grasp for the design and control of robotic manipulators. However, the effectiveness of such approach is dependent on the measurement tools that are available for use with human hands. While there are many sensing solutions that are designed for this purpose, obtaining a complete set of measurements of forces during grasp interaction is still challenging. In this work we aim to bridge this gap by introducing ExoSense, a passive hand exoskeleton. This device can provide position and orientation of the fingertips and, when integrated with the fingertip wearable force/torque sensing system ThimbleSense, a complete characterization of manipulation in terms of generalized forces and position of contacts on each fingertip in a completely wearable and unconstrained manner. After validating the device in terms of end-effector posture measurements and overall accuracy of grasp measurements, we report on a preliminary experiment aiming to show the potentialities of the system to study human internal grasp force variations and for neuroscientific investigation in general.
\end{abstract}

\section{INTRODUCTION}

As humans, we are capable of complex manipulation tasks, thanks to the extraordinarily bio-mechanic and sensorymotor architecture of our hands. Although trying to artificially replicate such an architecture would be clearly unfeasible, there is convincing evidence that valuable information, both kinematic and force related, can be extracted from human hand behavior to inform the design, control and sensing of artificial systems [1]. Information obtained from postural measurements, in particular the coordinated interfinger kinematic behavior described in terms of synergies, can be used to devise simplified and effective design guidelines for robotic hands [2]. The study of human force control can also offer interesting insights in terms of robotic manipulation. For example, [3] shows a bio-inspired sensing setup for robotic hands that aims to detect mechanical transients during grasp, while [4] uses a similar approach on grippers endowed with pressure arrays aiming to mimic capabilities of human tactile afferents. In [5] a five-fingered robotic hand is presented, inspired by force measurements obtained from humans grasping and lifting sensorized cylinders of different weights.

This work was partially supported by the European Community funded projects SOFTPRO and SOMA (contracts 688857 and 645599, respectively), by the ERC Advanced Grant no. 291166 SoftHands.

E.B., M.B. and A.B. authors are within Centro di Ricerca "E. Piaggio", University of Pisa, 56126 Pisa, Italy e.battaglia, matteo.bianchi, bicchicentropiaggio.unipi.it

M.G.C.,G.G. and A.B. are within the Soft Robotics for Human Cooperation and Rehabilitation research line, Istituto Italiano di Tecnologia, 30 Via Morego, 16163 Genova, Italy manuel.catalano, giorgio.grioli, antonio.bicchidit.it

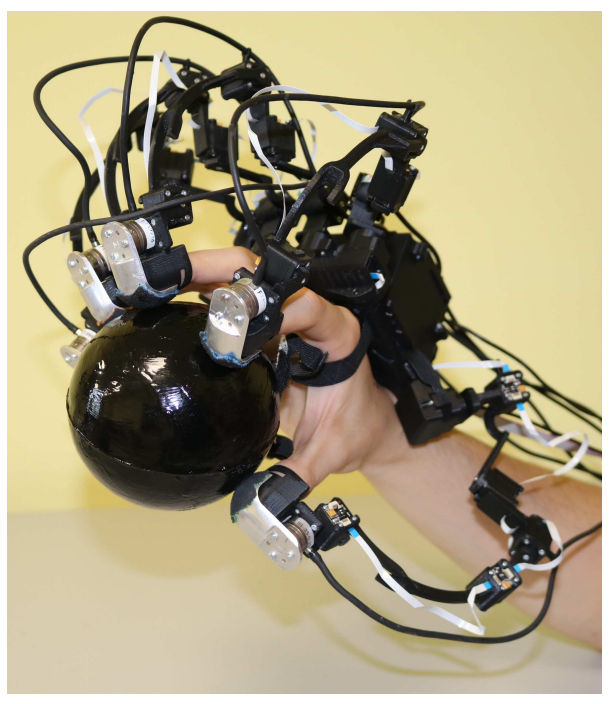

Fig. 1: ExoSense.

Study of grasp in robotics is traditionally done by a complete mathematical description of fingertip interaction with the grasped object [6]. A description of human grasp in the same terms would help to translate biology results in technological implementations: this however requires an exhaustive description of force and torque exerted by fingers and contact points on the grasped objects. There are not many wearable tools in the literature that can provide this kind of measurements: typical solutions for force sensing in hands use gloves endowed with tactile sensors [7], [8], [9]. While this represents a useful way to obtain global information on the tactile interaction, it cannot provide the complete information that was mentioned above. Different approaches can also be taken, e.g. [10] showed that visionbased force sensing could be feasible, while [11] uses an interesting approach that estimates forces on the fingertip from fingernail imaging techniques. However, to the best of our knowledge, there are no tools to retrieve contact points and force information in a wearable manner. To address this gap in [12] we introduced the ThimbleSense, a wearable fingertip sensing system that relies on force/torque measurements to obtain the location of contacts through the intrinsic tactile sensing [13] algorithm.

The approach proposed in [13] presented two limitations: (i) wearing rigid shells on the fingertip causes a distortion of the tactile sensation, and (ii) a motion capture system with LEDs was used to track position and orientation of the fingertips. Regarding (i), this is a limitation that is intrinsic in the approach chosen: in [12] an evaluation of its effect was done, and it was considered a reasonable trade off to 


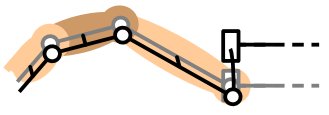

(a) Following the finger.

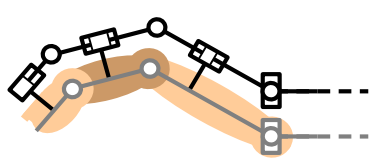

(b) Close parallel with sliding joints.

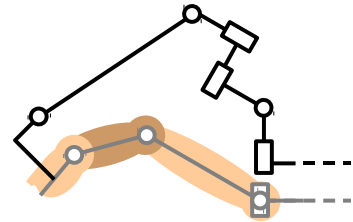

(c) Far parallel 6 d.o.f kinematic chain (used for the thumb).

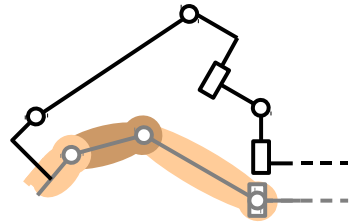

(d) Far parallel 5 d.o.f. kinematic chain (used for the fingers).

Fig. 2: ExoSense concept: possible solutions.

be able to obtain the complete set of measurements that the system can provide. For what concerns instead (ii), it would be desirable to have a system that is completely wearable, to allow an ecological investigation of human behavior. In this work we aim to resolve this problem. A common wearable approach to retrieve fingertip pose is to endow a glove with sensors, e.g. goniometers [14], [15], [16] or inertial measurement units [17], [18]. This has the advantage of being able to track movements with limited obstruction for the user, however it can run into problems when it comes to fitting (for example, goniometers need to be placed over joints to be effective, which makes the approach difficult to adapt to different sizes) and can be obtrusive. Other approaches (e.g. marker based [19], [20] or vision based [21], [22], [23]) rely on external sensors and would not be suitable for a self-contained wearable system.

The approach that we chose to pursue is to integrate ThimbleSense with a passive hand exoskeleton. Design of hand exoskeletons is a popular topic in literature, and there are a few that can provide measurement of hand kinematics as well as haptic feedback (e.g. [24], [25], [26], [27]): however, these systems are actuated, and usually designed for use in rehabilitation, and are not meant to be used primarily as a measurement tool to study grasp. Our idea, inspired by [27], is to present a passive exoskeleton (named ExoSense) that enables for the first time to get a complete force characterization in a wearable way. Furthermore, this approach could also provide a tool for a combined kinematic and force characterization, enabling to study synergistic patterns of these in a combined manner. Finally, ExoSense, thanks to its design and wearability, could be used as an input device to enable control of robotic hands in teleoperation.

\section{Problem Statement}

To build a device to obtain a force characterization of the human hand during fingertip grasping and manipulation tasks, one must combine a system capable of reconstructing the force and torque exchanged between each finger and the object, with a method of localizing the position and orientation of the fingertips in space. This paper deals with the problem of designing, implementing, calibrating and testing a solution for the latter, which is integrated with a wearable force/torque sensor. Considerations on overall system wearability led us to the choice of realizing the device in the form of an exoskeleton.

Given the five fingers of the human hand, each finger can be regarded as a chain of three links, with an Universal joint (U) between the first phalanx and the palm, and with two rotational joints $(\mathrm{R})$ between the first and second and between the second and third phalanges - i.e. an URR kinematic chain. The thumb makes an exception to this model, being more similar to an UUR kinematic chain or, following some sources, a SUR chain (i.e. with the first joint being Spherical).

The most straightforward approach for the implementation of an exo-skeletal solution consists in mimicking the kinematics of the finger and attaching the components of the exoskeleton to the phalanges of the fingers, one by one. A simple way to do this is depicted in Fig. 2 (a). However, this solution has the drawback of placing the exoskeleton on the sides of the fingers, potentially hindering the natural motions of the finger themselves. Moreover, with this approach it is critical to copy the finger kinematics exactly. This, in turn, can be a problem because of the extreme variability in sizes of hands among different subjects, and even more because of the inter-subject variability of the effective kinematics of the hand (e.g. see [28], [29]). Indeed, the rotational joints of the fingers are not completely parallel to the palm plane nor orthogonal to the finger main axis, but are slightly slanted of a different angle for every person. Looking closer, they are not pin joint at all, but are more similar to rolling pairs.

To avoid dealing with part of these problems, there are approaches as the one shown in Fig. 2(b). On this solution the rotational joints of the exoskeleton are kept parallel to the joints of the finger by the addition of several (5) sliding joints to the structure, which makes the design much more complex. This solution has the advantage of being positioned above the finger rather than on its side, reducing the encumbrance, and is also capable of adapting to different fingers length. Nevertheless, it still has some problems when facing inclination of the finger rotational axes. Note that both solutions (a) and (b) in Fig. 2 have the potential of causing distress or pain to the user's hand due to misalignment of the rotation axes, thus need to include some compliance in the attachment between the hand and the exoskeleton itself.

Pushing to the extreme consequences the ideas of solution (b), we can totally decouple not only the intermediate joints but also the links of the finger and exoskeleton and realize the exoskeleton in the form of an independent parallel kinematic chain, attached to the finger only by the fingertip. This solution, inspired by [27], where a similar design was implemented for a three-finger actuated exoskeleton, reduces the coupling between the exoskeleton and the finger, hence 
also the encumbrance and the user's discomfort. The main drawback of this solution is the difficulty of reconstructing the full configuration of the fingers, because it must be based on some model of the finger - either calibrated offline or estimated online - but this is not a problem for our application, where only the position of the fingertip is needed. This can be reconstructed simply from the forward kinematics of the exoskeleton. For these reasons, in this paper we adopt this solution and extend it to the whole hand.

\section{DESIGN}

Following the approach described in the previous section, we developed the exoskeleton as a series of kinematic chains attached to a common base. We used the six degrees of freedom (d.o.f.) solution shown in Figure $2 \mathrm{c}$ for the thumb, which is more complex than the fingers [30], while the five d.o.f. shown in Figure 2d was preferred for the fingers. In this section we describe the final CAD model of ExoSense, as well as the hardware and software used with it.

\section{A. CAD model}

Figure 3 shows an overview of the CAD model and its components. As mentioned earlier the kinematic chains are composed of six joints for the thumb and five for the fingers, and a ThimbleSense is assembled at the end of each chain. The kinematic chains are identical for index, middle and ring finger, while the little finger has a kinematic chain with the same joint structure but different size of the links. The inner shells of each ThimbleSense were replaced with a custom designed shell that can be assembled to the last link of ExoSense. These custom shells have each a slot that is used in the calibration phase. The thumb kinematic chain also has an element that is used to lock in place the first degree of freedom, and which is removed after the calibration phase.

The magnetic encoders (As5045 from Austria microsystem, $\pm 0.01 \mathrm{deg})$ are assembled in a similar manner on each joint, as shown in the detailed view in Figure 3, and are connected to each other and to the boards through standard FFC connectors. A separate board is used for each finger, and all five of them are located in the box, which is assembled to the base representing the starting point of all five kinematic chains. All the electronic boards used in the device were developed under the Natural Machine Motion Initiative (more information is available in [31]).

An InvenSense MPU-9250 IMU is placed on the box, connected to its own board. The five encoder boards are connected in daisy chain and are then plugged to a laptop through a USB cable; the IMU board is instead connected directly to a laptop through USB. Finally, the ATI nano 17 Force/Torque sensors [32] used by the ThimbleSenses are connected to a switch through ethernet and data from them is fed to the laptop by an ethernet cable. The overall sampling frequency of the system is $12 \mathrm{~Hz}$. The structural components of the exoskeleton are all 3D printed and built in ABS.

To have correct readings from the encoders a calibration procedure is needed. This is obtained using of a suitable calibration structure (Figure 4a), which can also be employed to store the exoskeleton safely when it is not being used.

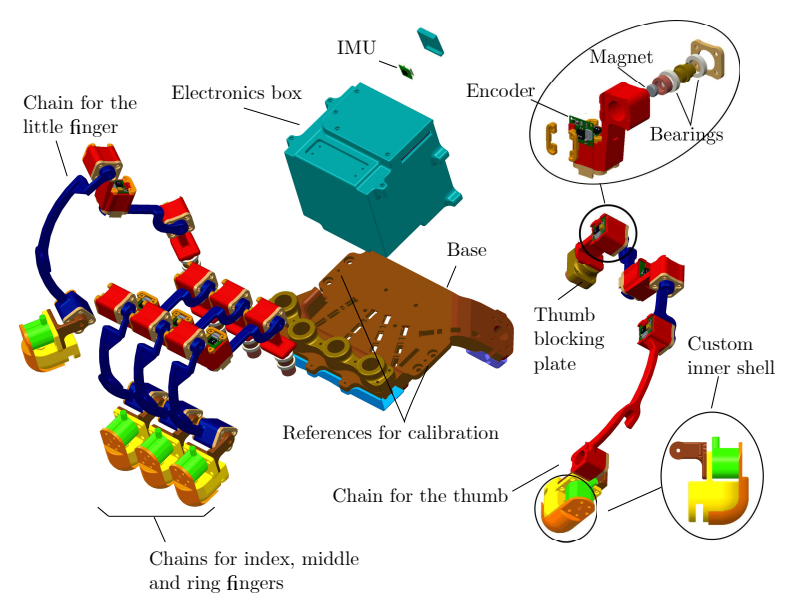

Fig. 3: ExoSense CAD model and components.

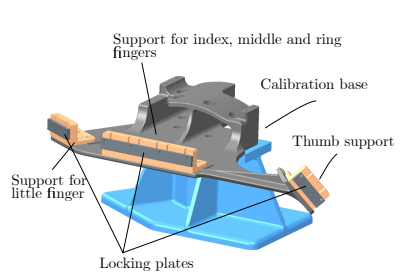

(a) CAD model of the calibration structure.

Fig. 4: ExoSense calibration and physical prototype.

Proper reference for each end effector is obtained through supports placed on the structure, each having a slot corresponding to a negative of the inner shell of a ThimbleSense. To ensure proper placement of the shells on the structure, blocking plates are assembled on each inner shell through their open slots, and they are fixed on the calibration structure with screws, blocking the system completely. Calibration is then performed by setting the zeros of the encoders on each board. Figure 4 shows the physical prototype assembled on the calibration structure: this is the zero configuration of ExoSense, which will be used as a reference for all data acquisitions.

\section{B. Software}

To have feedback on the performance of ExoSense, a visualization tool was deemed necessary. ThimbleSense used MATLAB as a visualization interface, by importing the CAD model and showing contact points and forces applying on them: however, owing to the much more complex structure of ExoSense, this solution would not work well in this case, since the higher number of elements would slow down rendering and cause lag when attempting to do visualization while also reading the sensors.

For this reason, while we will still be using MATLAB for post processing and analysis of data, we decided to instead visualize our results during acquisition with Blender [33]. Blender is a free and open source 3D creation suite, that can also be interfaced with sensors and used as a virtual reality 


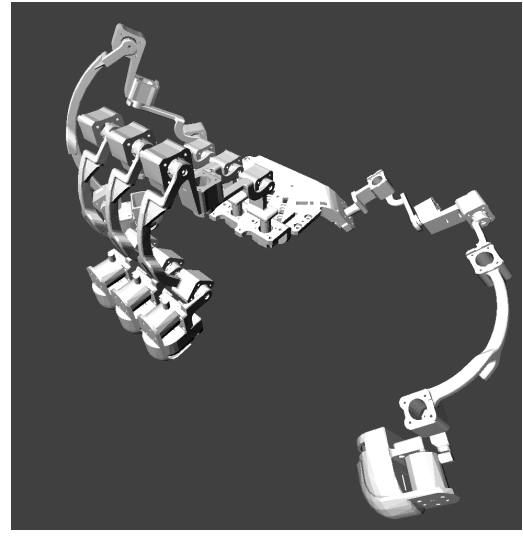

(a) Reconstruction of ExoSense in the virtual environment.

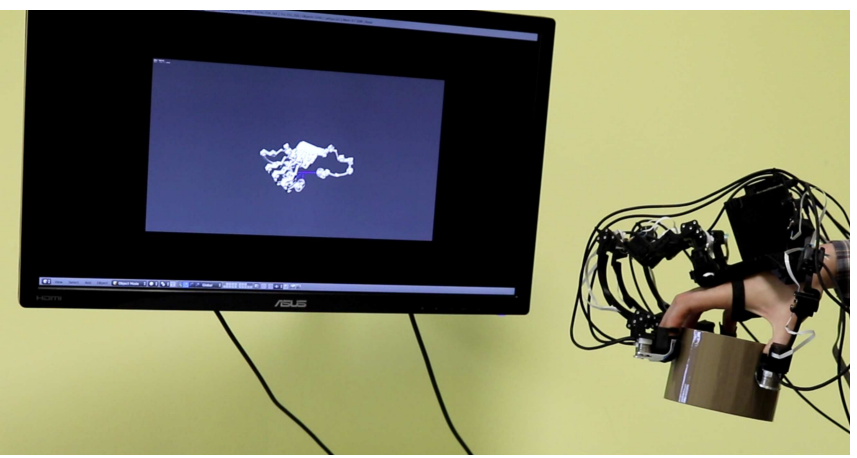

(b) Example of use of the virtual reality environment.

Fig. 5: Visualization.

environment. The CAD model of each link was imported in Blender, and the kinematic chain defined based on geometry information obtained from the CAD. The electronic boards, which come with their own $\mathrm{C}++$ code that can be used to obtain data from them, were then connected to the software by compiling the $\mathrm{C}++$ code into Python modules through Cython [34]. Since Blender supports use of Python, this made it possible to read all sensors directly in Blender. A similar procedure was used to read the ATI sensors by building their own $\mathrm{C}++$ libraries in a Python module.

The encoder readings were fed to the Blender game engine which calculated the forward kinematic to visualize the movement, while the data from the IMU was used in a passive complementary filter [35] to estimate orientation of the base, which is used for visualization purposes and to know the direction of gravity. Knowledge of the forward kinematics makes it possible to know position and orientation of each ThimbleSense, which are rigidly attached to the end effectors of each finger. Position of contacts on the ThimbleSense shells is also estimated by using the intrinsic tactile sensing algorithm described in [13], and contact centroids as well as forces are visualized.

\section{VALIDATION}

In this section we describe a validation procedure aiming to evaluate the performance of the ExoSense. This validation is composed of two distinct phases: first posture estimation of the end effector is evaluated by assembling ExoSense on a modified calibration structure; then an overall validation of the grasp measurement is performed by comparing the measured wrench to a ball of known weight, and by verifying a lack of variation of internal forces while squeezing the same ball.

\section{A. Posture estimation}

For the first step we designed a new set of supports to be placed on the calibration structure, by inserting an extrusion of $20 \mathrm{~mm}$ between the base and the interface with the shells for the supports used for calibration (Figure 6a). ExoSense was then placed on this new structure and data from the encoders was recorded through the Blender interface.

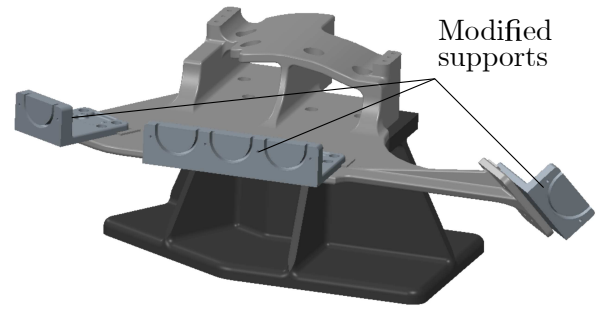

(a) CAD model of the modified calibration structure for evaluation of posture estimaton accuracy.

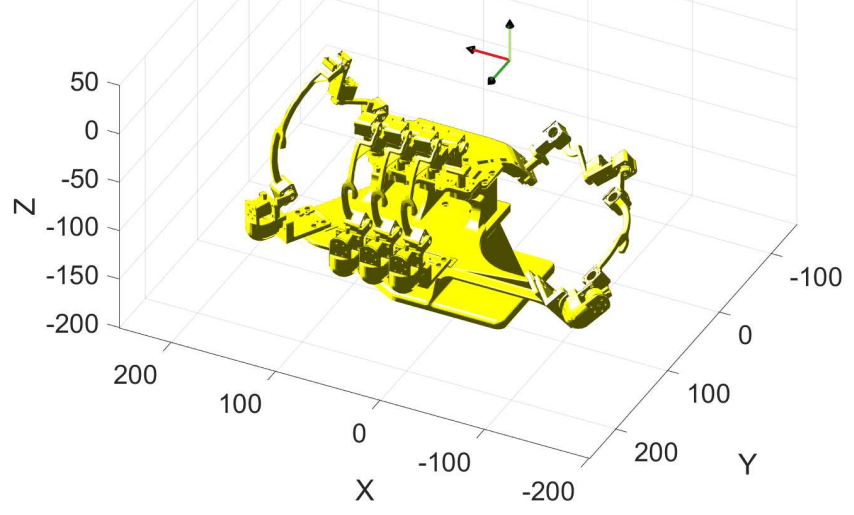

(b) Posture reconstruction on MATLAB.

Fig. 6: Posture estimation.

\begin{tabular}{cccccc}
\hline Components & Thumb & Index & Middle & Ring & Little \\
\hline$x[\mathrm{~mm}]$ & 0.917 & 2.002 & 2.429 & 3.106 & 0.0445 \\
$y[\mathrm{~mm}]$ & 1.239 & 0.554 & 0.083 & 0.397 & 1.064 \\
$z[\mathrm{~mm}]$ & 0.114 & 0.669 & 0.756 & 0.476 & 0.0432 \\
roll [deg] & 3.835 & 0.039 & 0.0490 & 0.0656 & 0.0631 \\
pitch [deg] & 3.771 & 1.208 & 0.1370 & 0.279 & 2.395 \\
yaw [deg] & 0.872 & 0.256 & 0.0917 & 0.427 & 0.411 \\
\hline
\end{tabular}

TABLE I: RMSE for position components and roll, pitch and yaw, for each finger.

Figure 6b shows a reconstruction obtained in MATLAB, from which a visual check shows that the end effectors appear placed correctly on the new structure. Table [ ] shows 
a more in depth quantitative analysis of the error, obtained as a difference between the position and orientation of the end effector as known by the CAD in the new validation structure, and the same quantities as measured by the forward kinematic calculated by the encoder. Both are expressed with respect to the base reference frame shown in Figure 6b. The quantitative results show an error of a few millimeters/degrees respectively, which substantially confirms the first visual impression.

\section{B. Complete grasp validation}

The second phase of the validation aims to evaluate the overall performance of ExoSense when it comes to measuring grasp, considering all measurements (encoder readings, orientation estimation from the IMU, force/torque measurements and contact point estimation) simultaneously. We followed a procedure similar to the one used in [12], relying on the framework presented in [6].

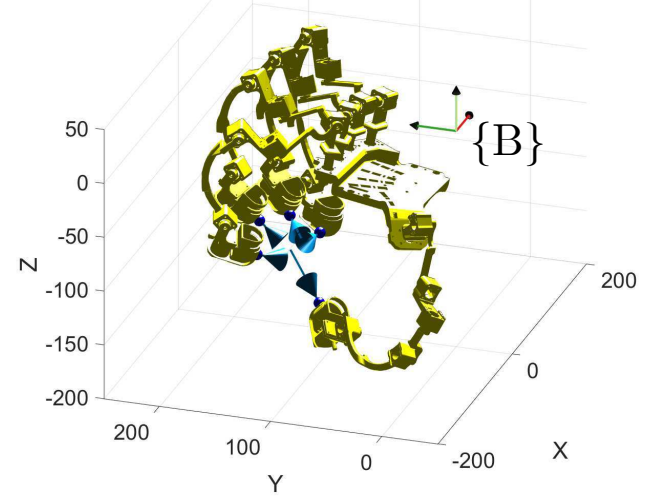

Fig. 7: Grasp validation: reconstruction of grasp.

More specifically, a sponge ball (mass $m_{1}=0.053 \mathrm{Kg}$, radius $R_{1}=50 \mathrm{~mm}$ ) was grasped by a subject (male, age 29) while wearing ExoSense, using all five fingers $\left(n_{f}=5\right)$ to interact with the object. The task was a simple lift and hold of the ball. Validation studied the data registered during the experiment, by first checking that the grasp equilibrium condition was verified during the holding phase, and then seeing if the internal force variation was inside the null space of the grasp matrix. Figure 7 shows a reconstruction of the grasp in MATLAB, as well as the base reference frame $\{B\}$ respect to which all links postures are calculated.

To verify equilibrium, we needed to check that the vector of generalized forces $\mathbf{t} \in \mathbb{R}^{6 n_{f}}$, as measured from ExoSense, was coherent with the applied external weight wrench $\mathbf{w} \in \mathbf{R}^{6}$ as described by the grasp equation

$$
\mathbf{w}=G \mathbf{t}
$$

which holds under quasi static conditions. The grasp matrix $G \in \mathbb{R}^{6 \times 6 n_{f}}$ can be obtained as

$$
G=\left(\begin{array}{ccccc}
I_{3} & 0_{3 \times 3} & \cdots & I_{3} & 0_{3 \times 3} \\
\wedge \mathbf{c}_{1} & I_{3} & & \wedge \mathbf{c}_{n_{f}} & I_{3}
\end{array}\right)
$$

where $^{\wedge}(\cdot)$ is the skew-matrix operator and $\mathbf{c}_{1} \ldots \mathbf{c}_{n_{f}}$ are the contact points coordinates calculated through the intrinsic tactile sensing algorithm [13], expressed in a reference system with origin in the center of mass of the ball $\mathbf{b}$ and oriented as the fixed base frame $\{B\}$.

The position of the center of mass of the ball is not measured exactly by the system; however, since we can reasonably assume that the center of mass is the same as the geometric center, we can estimate its position from the contact centroid algorithm. More specifically, an estimate $\mathbf{b}_{i}$ of the ball center can be obtained as

$$
\mathbf{b}_{i}=\mathbf{c}_{i}+R \mathbf{n}_{i},
$$

where $R$ is the radius of the ball in $\mathrm{mm}$ and $\mathbf{n}_{i}$ is the unit vector normal to the contact surface during grasp, which can be obtained from the intrinsic tactile algorithm. The ball center position estimate $\mathbf{b}$ can then be obtained as

$$
\mathbf{b}=\frac{1}{n_{f}} \sum_{i=1}^{n_{f}} \mathbf{b}_{i} .
$$

where $n_{f}=5$ is the number of fingers in contact. We now have everything we need to verify goodness of measurements and can compute the wrench error as

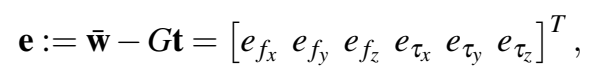

where $\mathbf{w}=\overline{\mathbf{w}}=[0,0,-m g, 0,0,0]^{T}$, with $g=9.81 \mathrm{~m} / \mathrm{s}^{2}$, is the weight wrench which is known from knowing the mass of the ball.

\begin{tabular}{cccc}
\hline Components & $f_{x}$ & $f_{y}$ & $f_{z}$ \\
\hline RMSE & $0.11[\mathrm{~N}]$ & $0.12[\mathrm{~N}]$ & $0.14[\mathrm{~N}]$ \\
Percentage RMSE & $2.07 \%$ & $2.29 \%$ & $2.72 \%$ \\
\hline Components & $\tau_{x}$ & $\tau_{y}$ & $\tau_{z}$ \\
RMSE & $22.7[\mathrm{~N} \mathrm{~mm}]$ & $22.0[\mathrm{~N} \mathrm{~mm}]$ & $29.0[\mathrm{~N} \mathrm{~mm}]$ \\
\hline
\end{tabular}

TABLE II: RMSE for the grasp equilibrium condition (error wrench e).

Figure $8 \mathrm{a}$ is a double plot showing the evolution of norms of forces on the fingertips and estimated ball position, respectively. A transitory effect is noticeable at the beginning while the ball is being grasped, and is followed by a time interval where the ball position is more stable: this time frame is shown by the two dashed lines in the plot showing the ball position. This is the portion of the task where quasi static conditions have been considered to hold and will be used for the rest of the analysis.

Figure $8 \mathrm{~b}$ shows the overall wrench error. From the zoomed in detail it can be seen be seen that the force error overs between $\pm 0.2 \mathrm{~N}$ in the stable contact range, while the torque error is between $\pm 30 \mathrm{~N} \mathrm{~mm}$. Figure $8 \mathrm{~d}$ shows the percentage error, obtained by normalizing the force components of e respect to the norm of force measured on the thumb, as was done in [12]. In can be seen that the error is in the $\pm 4 \%$ range. Table II quantifies the performance more accurately by showing root mean square errors (RMSE) for force and torque components. The results appear to be comparable respect to what was observed in [12], with some quantities showing a better performance. 

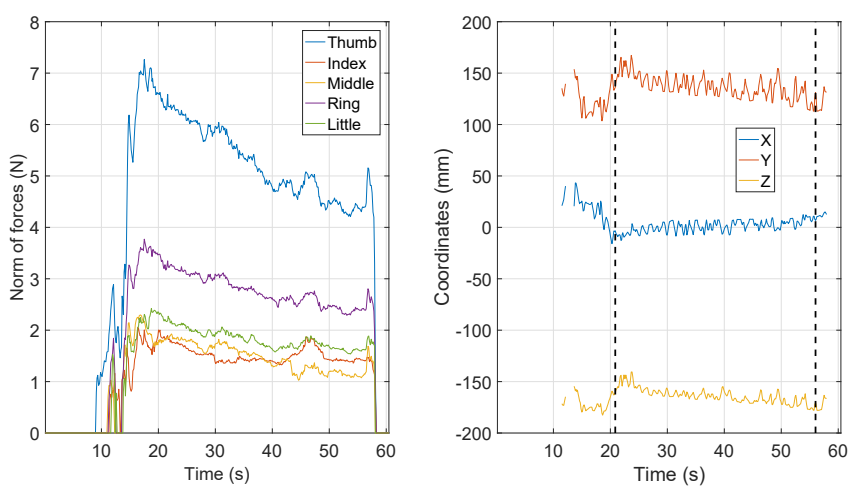

(a) Forces on fingertips and estimated ball position with respect to the base frame.

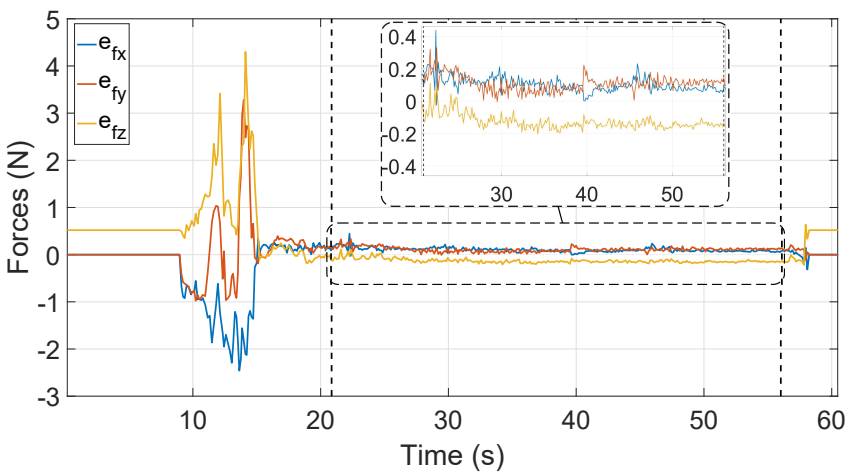

(b) Force error.

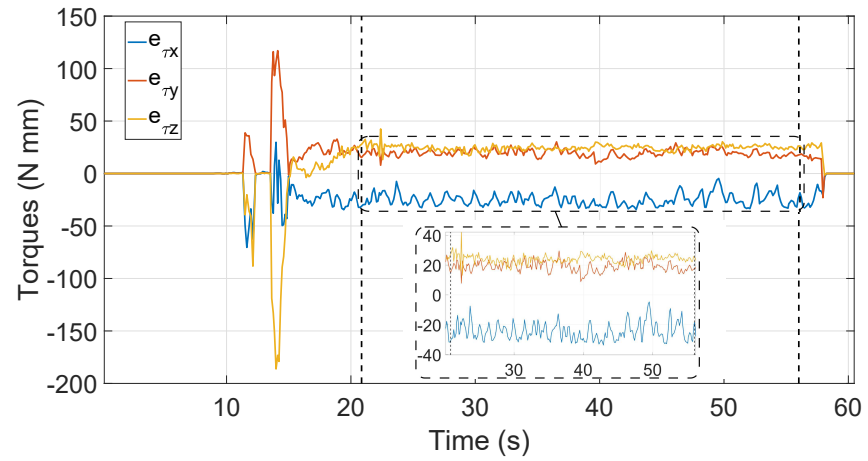

(c) Torque error.

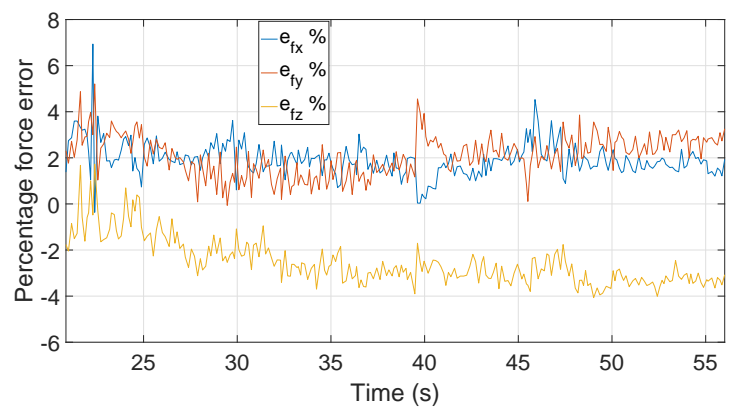

(d) Percentage force error.

Fig. 8: Grasp validation: forces, position of the grasped sphere and percentage force error.
We now proceed to studying internal forces variation during the task. Let us consider $\Delta \mathbf{t}=\mathbf{t}_{k}-\mathbf{t}_{0}$, where $\mathbf{t}_{0}$ is the generalized force measured at the initial sample $s_{0}$ (corresponding to the first sample of the stable zone, e.g. first dashed line in Figure $8 \mathrm{a}$ and $\mathbf{t}_{k}$ is the force measured in a generic later sample $s_{k}$. Since the ball is held still, the external wrench $\mathbf{w}$ is not changing, and from $\mathbf{w}=G \mathbf{t}_{0}=$ $G \mathbf{t}_{k} \forall k$ follows that the vector $\Delta \mathbf{t}$, non-null for variation of forces, lies in the null space of $G(\Delta \mathbf{t} \in \mathscr{N}(G))$. It is known from grasp theory and linear algebra ([6], Meyer [36]) that $\left(I-G^{+} G\right)$, where $I$ is the identity matrix, is a projector to $\mathscr{N}(G)$. Therefore, if we compute

$$
\Pi_{G}(\Delta \mathbf{t}):=\left(I-G^{+} G\right) \Delta \mathbf{t}=\left[I-G^{T}\left(G G^{T}\right)^{-1} G\right] \Delta \mathbf{t}
$$

since $\Delta \mathbf{t} \in \mathscr{N}(G)$ it should be true that $\Pi_{G}(\Delta \mathbf{t})=\Delta \mathbf{t}$. We can then define the error

$$
\begin{aligned}
& \mathbf{e}_{\Pi}:=\Pi_{G}(\Delta \mathbf{t})-\Delta \mathbf{t}=
\end{aligned}
$$

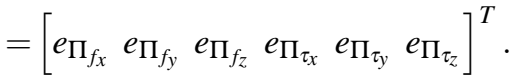

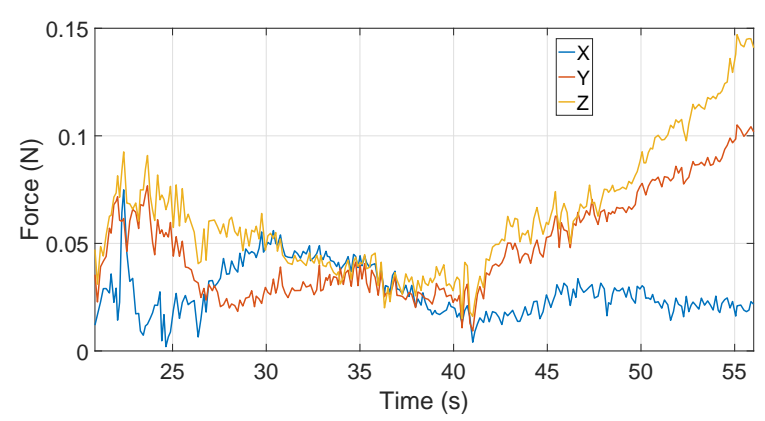

Fig. 9: Internal force projection error.

\begin{tabular}{cccccc}
\hline Components & Thumb & Index & Middle & Ring & Little \\
\hline$f_{x}[\mathrm{~N}]$ & 0.0543 & 0.0151 & 0.0294 & 0.0162 & 0.0155 \\
$f_{y}[\mathrm{~N}]$ & 0.0991 & 0.0238 & 0.0424 & 0.0431 & 0.0208 \\
$f_{z}[\mathrm{~N}]$ & 0.0980 & 0.0656 & 0.0772 & 0.0382 & 0.0521 \\
\hline
\end{tabular}

TABLE III: RMSE for the force components of $\mathbf{e}_{\Pi}$.

Figure 9 shows the evolution of this error for the force components, quantified in this plot as the RMSE of each component over all fingertips, for each sample. Table III shows the overall RMSE during the acquisition, for each component and each finger. Results are again comparable with respect to what was found in [12].

\section{PRELIMINARY EXPERIMENTS}

In this section we show a preliminary experiment that aims to show an interesting scenario where ExoSense could be used to study force behavior during grasp. Figure 10 shows the experimental setup: a larger ball (mass $64 \mathrm{~g}$, radius 50 $\mathrm{mm}$ ) was attached to a second ball, of mass $320 \mathrm{~g}$ and radius $33 \mathrm{~mm}$. The spheres were placed in a cylindrical container, with the smaller and heavier one laying below. The task consisted in grasping the larger one and lifting both up.

Figure 11a shows the evolution of forces. It can be noticed that the $\mathrm{Z}$ component of overall force measured by the 


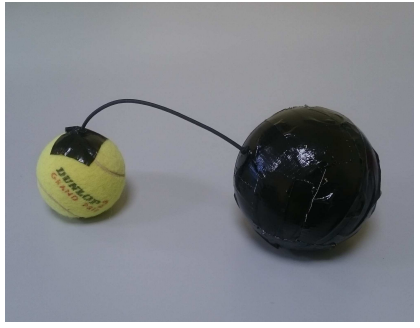

(a) Double sphere experimental setup.
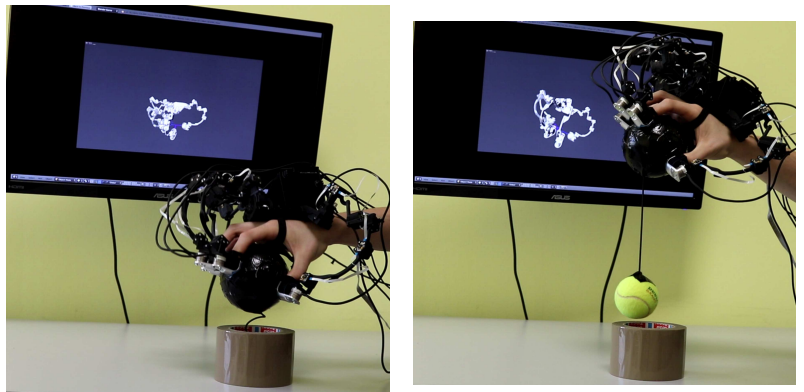

(b) Initially only the first sphere is lifted.

(c) The second one is also lifted from the table, leading to increased forces.

Fig. 10: Experimental setup for the final experiment.

ThimbleSenses increases first to a value slightly higher than the weight of the first ball (where the difference can be ascribed to the wire connecting the two), and then increases again when the second ball is lifted to the overall weight of the two balls (the lower dashed lines includes the weight of the wire). Figure $11 \mathrm{~b}$ shows the evolution of norms of forces on each fingertip: while the norms are clearly different, the evolution shows a similar behavior over the different fingers. To investigate this aspect, the mean value and standard deviation of each norm was calculated, for each fingertip, over the interval of time where the grasp was occurring, as individuated by inspection of force values (dashed vertical lines in Figure $11 \mathrm{~b}$. We then normalized each signal by subtracting the mean and dividing by the standard deviation. The result is shown in Figure $11 \mathrm{c}$; it can be seen that, once normalized, the evolution of forces for all fingers except the index is visually almost the same. Indeed, looking at the correlation values, correlation was found to be greater or equal than 0.94. Since only one subject was involved on a single trial, we cannot make any strong claims on this preliminary result: however, the implications in term of the possibility of force synergies are interesting, and we believe that further investigation in a more structure experimental protocol would contribute to the already existing research on the topic (e.g.[37]).

\section{CONCLUSIONS}

In this work we presented ExoSense, a passive hand exoskeleton designed to provide complete measurement of grasp, thanks to its integration with the fingertip wearable force/torque sensing system ThimbleSense and the usage of the intrinsic tactile sensing. ExoSense enables to retrieve the

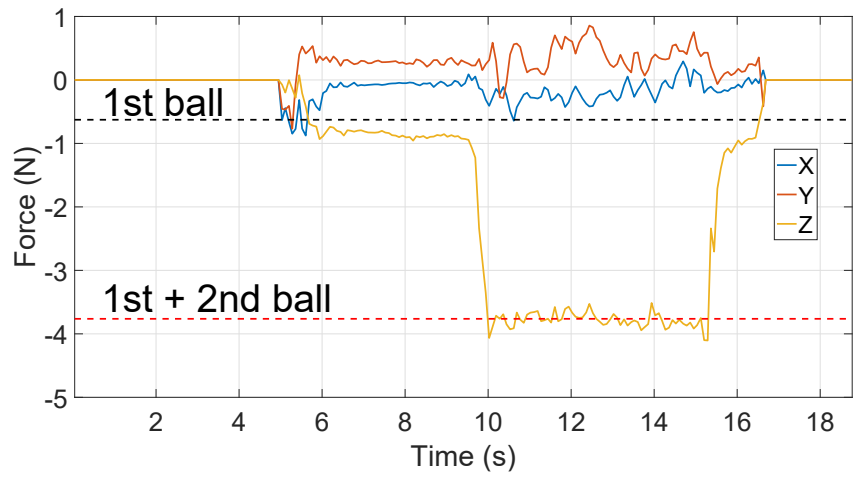

(a) Measured wrench and norm of forces during the double sphere task.

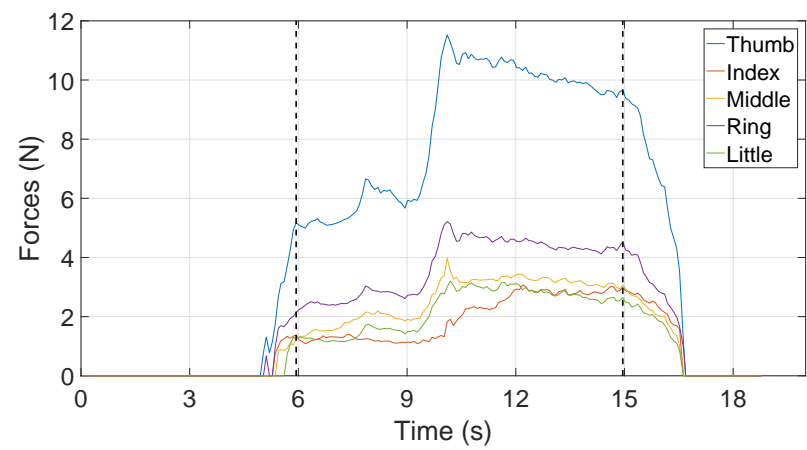

(b) Measured wrench and norm of forces during the double sphere task.

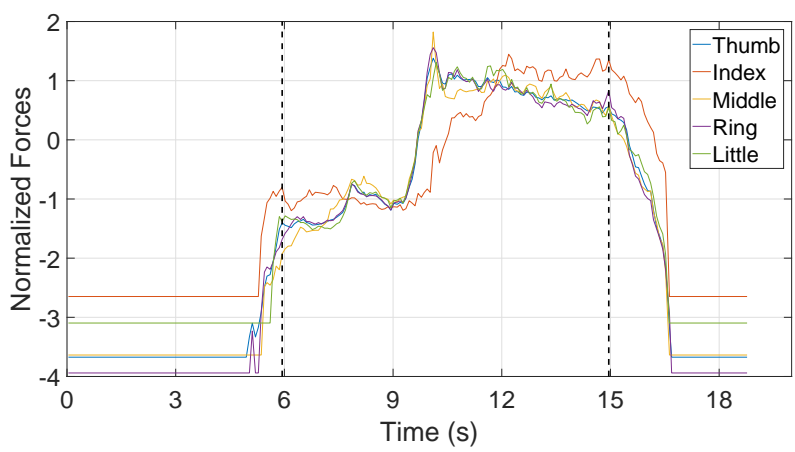

(c) Normalized norm of forces on fingertips.

Fig. 11: Experimental setup for the final experiment.

ThimbleSense pose in a global inertial frame, thus allowing, for the first time, a completely wearable way to characterize human grasping. We validated the device obtaining errors of $\approx 3 \mathrm{~mm}$ for position and $\approx 3 \mathrm{deg}$ for orientation. In terms of force and toque measurements, RMSE were respectively below $0.15 \mathrm{~N}(\approx 2.8 \%$ of thumb force norm) and $30 \mathrm{~N}$ $\mathrm{mm}$. We also showed a preliminary experiment whose goal was to show the potentialities of ExoSense in studying multi finger force variation at fingertips. These variations are a result of variation of external forces, showing interesting commonalities of norm of forces on each fingertip.

The findings ExoSense will enable to achieve could be then profitably translated for the force control of artificial end-effectors, in a mutual inspiration between robotics and neuroscience. At the same time, it could offer a portable 
solution to assess the outcomes of rehabilitative procedures. Future work will move in a twofold direction: on one hand we will continue to develop the device to make it lighter and less intrusive in terms of both size and weight, while on the other we will begin using this device to investigate human manipulation. Usage of the ExoSense as an input device to remotely control robotic hands in tele-operation will also be evaluated.

\section{ACKNOWLEDGMENT}

The authors gratefully acknowledge Giacomo Dinuzzi, Vinicio Tincani and Mattia Poggiani for their help with the design and assembly of the mechanical and electronical components of the exoskeleton.

\section{REFERENCES}

[1] M. Santello, M. Bianchi, M. Gabiccini, E. Ricciardi, G. Salvietti, D. Prattichizzo, M. Ernst, A. Moscatelli, H. Jörntell, A. M. Kappers, et al., "Hand synergies: integration of robotics and neuroscience for understanding the control of biological and artificial hands," Physics of life reviews, vol. 17, pp. 1-23, 2016.

[2] M. G. Catalano, G. Grioli, E. Farnioli, A. Serio, C. Piazza, and A. Bicchi, "Adaptive synergies for the design and control of the pisa/iit softhand," The International Journal of Robotics Research, vol. 33, no. 5, pp. 768-782, 2014.

[3] B. B. Edin, L. Ascari, L. Beccai, S. Roccella, J.-J. Cabibihan, and M. Carrozza, "Bio-inspired sensorization of a biomechatronic robot hand for the grasp-and-lift task," Brain research bulletin, vol. 75, no. 6, pp. 785-795, 2008.

[4] J. M. Romano, K. Hsiao, G. Niemeyer, S. Chitta, and K. J. Kuchenbecker, "Human-inspired robotic grasp control with tactile sensing," IEEE Transactions on Robotics, vol. 27, no. 6, pp. 1067-1079, 2011.

[5] Y. Kamikawa and T. Maeno, "Underactuated five-finger prosthetic hand inspired by grasping force distribution of humans," in Intelligent Robots and Systems, 2008. IROS 2008. IEEE/RSJ International Conference on. IEEE, 2008, pp. 717-722.

[6] A. Bicchi, "On the problem of decomposing grasp and manipulation forces in multiple whole limb manipulation," Int. Journal of Robotics and Autonomous Systems, vol. 13, no. 2, pp. 127 - 147, 1994.

[7] Q. Ye, M. Seyedi, Z. Cai, and D. T. Lai, "Force-sensing glove system for measurement of hand forces during motorbike riding," International Journal of Distributed Sensor Networks, vol. 11, no. 11, p. $545643,2015$.

[8] P.-C. Hsiao, S.-Y. Yang, B.-S. Lin, I.-J. Lee, and W. Chou, "Data glove embedded with 9-axis imu and force sensing sensors for evaluation of hand function," in Engineering in Medicine and Biology Society $(E M B C), 2015$ 37th Annual International Conference of the IEEE. IEEE, 2015, pp. 4631-4634.

[9] M. Bianchi, R. Haschke, G. Büscher, S. Ciotti, N. Carbonaro, and A. Tognetti, "A multi-modal sensing glove for human manualinteraction studies," Electronics, vol. 5, no. 3, p. 42, 2016.

[10] T.-H. Pham, A. Kheddar, A. Qammaz, and A. A. Argyros, "Towards force sensing from vision: Observing hand-object interactions to infer manipulation forces," in Proceedings of the IEEE Conference on Computer Vision and Pattern Recognition, 2015, pp. 2810-2819.

[11] T. R. Grieve, J. M. Hollerbach, and S. A. Mascaro, "Optimizing fingernail imaging calibration for $3 \mathrm{~d}$ force magnitude prediction," IEEE transactions on haptics, vol. 9, no. 1, pp. 69-79, 2016.

[12] E. Battaglia, M. Bianchi, A. Altobelli, G. Grioli, M. G. Catalano, A. Serio, M. Santello, and A. Bicchi, "Thimblesense: a fingertipwearable tactile sensor for grasp analysis," IEEE transactions on haptics, vol. 9, no. 1, pp. 121-133, 2016.

[13] A. Bicchi, J. K. Salisbury, and D. L. Brock, "Contact sensing from force measurement," The International Journal of Robotics Research, vol. 12, pp. 249-262, 1993.

[14] N. Williams, J. Penrose, C. Caddy, E. Barnes, D. Hose, and P. Harley, "A goniometric glove for clinical hand assessment: construction, calibration and validation," Journal of Hand Surgery, vol. 25, no. 2, pp. 200-207, 2000.

[15] P. Kumar, J. Verma, and S. Prasad, "Hand data glove: a wearable realtime device for human-computer interaction," International Journal of Advanced Science and Technology, vol. 43, 2012.
[16] A. Tognetti, F. Lorussi, G. Dalle Mura, N. Carbonaro, M. Pacelli, R. Paradiso, and D. De Rossi, "New generation of wearable goniometers for motion capture systems," Journal of neuroengineering and rehabilitation, vol. 11, no. 1, p. 56, 2014.

[17] T. L. Baldi, M. Mohammadi, S. Scheggi, and D. Prattichizzo, "Using inertial and magnetic sensors for hand tracking and rendering in wearable haptics," in World Haptics Conference (WHC), 2015 IEEE. IEEE, 2015, pp. 381-387.

[18] T. Mańkowski, J. Tomczyński, and P. Kaczmarek, "Cie-dataglove, a multi-imu system for hand posture tracking," in International Conference Automation. Springer, 2017, pp. 268-276.

[19] Q. Fu and M. Santello, "Tracking whole hand kinematics using extended kalman filter," in Engineering in Medicine and Biology Society (EMBC), 2010 Annual International Conference of the IEEE. IEEE, 2010, pp. 4606-4609.

[20] M. Gabiccini, G. Stillfried, H. Marino, and M. Bianchi, "A datadriven kinematic model of the human hand with soft-tissue artifact compensation mechanism for grasp synergy analysis," in Intelligent Robots and Systems (IROS), 2013 IEEE/RSJ International Conference on. IEEE, 2013, pp. 3738-3745.

[21] A. Erol, G. Bebis, M. Nicolescu, R. D. Boyle, and X. Twombly, "Vision-based hand pose estimation: A review," Computer Vision and Image Understanding, vol. 108, no. 1, pp. 52-73, 2007.

[22] F. Weichert, D. Bachmann, B. Rudak, and D. Fisseler, "Analysis of the accuracy and robustness of the leap motion controller," Sensors, vol. 13 , no. 5 , pp. $6380-6393,2013$.

[23] P. Panteleris and A. Argyros, "Back to rgb: $3 d$ tracking of hands and hand-object interactions based on short-baseline stereo," arXiv preprint arXiv:1705.05301, 2017.

[24] P. Garrec, J. Friconneau, Y. Measson, and Y. Perrot, "Able, an innovative transparent exoskeleton for the upper-limb," in Intelligent Robots and Systems, 2008. IROS 2008. IEEE/RSJ International Conference on. IEEE, 2008, pp. 1483-1488.

[25] Z. Ma, P. Ben-Tzvi, and J. Danoff, "Sensing and force-feedback exoskeleton robotic (safer) glove mechanism for hand rehabilitation," in ASME 2015 international design engineering technical conferences and computers and information in engineering conference. American Society of Mechanical Engineers, 2015, pp. V05AT08A036V05AT08A036.

[26] P. Ben-Tzvi, J. Danoff, and Z. Ma, "The design evolution of a sensing and force-feedback exoskeleton robotic glove for hand rehabilitation application," Journal of Mechanisms and Robotics, vol. 8, no. 5, p. 051019, 2016.

[27] I. Sarakoglou, A. Brygo, D. Mazzanti, N. G. Hernandez, D. G. Caldwell, and N. G. Tsagarakis, "Hexotrac: A highly under-actuated hand exoskeleton for finger tracking and force feedback," in Intelligent Robots and Systems (IROS), 2016 IEEE/RSJ International Conference on. IEEE, 2016, pp. 1033-1040.

[28] M. Grebenstein, M. Chalon, G. Hirzinger, and R. Siegwart, "A method for hand kinematics designers 7 billion perfect hands," 2010.

[29] I. Kapandjy, The Physiology of the Joints, vol. I - Upper Limb. Churchill Livingstone, 2009.

[30] M. Bianchi, P. Salaris, and A. Bicchi, "Synergy-based hand pose sensing: Reconstruction enhancement," The International Journal of Robotics Research, vol. 32, no. 4, pp. 396-406, 2013.

[31] C. Della Santina, C. Piazza, G. M. Gasparri, M. Bonilla, M. G. Catalano, G. Grioli, M. Garabini, and A. Bicchi, "The quest for natural machine motion: An open platform to fast-prototyping articulated soft robots," IEEE Robotics \& Automation Magazine, vol. 24, no. 1, pp. 48-56, 2017.

[32] ATI. F/t sensor: Nano17. [Online]. Available: http://www.ati-ia.com/ products/ft/ft_models.aspx?id=Nano17

[33] B. Foundation. Blender. [Online]. Available: https://www.blender.org/

[34] Cython. [Online]. Available: http://cython.org/

[35] G. Santaera, E. Luberto, A. Serio, M. Gabiccini, and A. Bicchi, "Lowcost, fast and accurate reconstruction of robotic and human postures via imu measurements," in Robotics and Automation (ICRA), 2015 IEEE International Conference on. IEEE, 2015, pp. 2728-2735.

[36] C. D. Meyer, Matrix Analysis and Applied Linear Algebra. Philadelphia, PA: Society for Industrial and Applied Mathematics, 2000.

[37] M. Santello and J. F. Soechting, "Force synergies for multifingered grasping," Experimental Brain Research, vol. 133, no. 4, pp. 457 467, 2000. 